\title{
Pathogenesis of acute appendicitis: review
}

\begin{abstract}
Based on the analysis of literature and our own studies we propose hypothesis of the pathogenesis of acute appendicitis (AA), which differs from the generally accepted by following provisions. Acute appendicitis develops as a result of immunological reaction and hyperplasia of the lymphoid tissue of the appendix. The frequency of AA correlates with the rapid growth of sex hormones and depends on age, gender and time of year. Only a small percentage of primary hyperplasia causes a complete occlusion of the lumen and destructive AA. Usually it is exposed to regression without causing the typical symptoms but leaving the damaged nervous system and/or sclerotic changes that violate the peristalsis of the appendix. Feces, lingering in appendix eventually harden, increasing in size and often are calcified. In one of the subsequent attacks of lymphoid hyperplasia, the walls of the appendix wrap around over fecalith, causing obstruction of the lumen, the formation of a closed cavity and the known mechanisms of inflammation. The formation of a closed cavity with high pressure leads to reflex strengthening of tone of the digestive tract, which explains the pain in the epigastrium and vomiting in the first hours of the disease. This is accompanied by increased of the anal canal pressure. The inflammatory process in the appendix leads to increased tone especially in segments of the intestine, lying next to it. Which manifests in the form of spasm of the sigmoid colon and/or ileum, as well as the shortening of the dome of the cecum or/and as the concavity of its inner wall. An increase in the tone of the digestive tract is a nonspecific reaction to inflammation.
\end{abstract}

Keywords: acute appendicitis, appendix, pathogenesis, children, diagnosis
Volume 10 Issue 6 - 2019

Michael D Levin

Department of Pediatric Radiology of the I-st State Hospital, Belarus

Correspondence: Michael D Levin, Department of Pediatric Radiology of I-st Hospital, Minsk, Belarus; State Geriatric Center, Netanya, Israel, Email nivel70@hotmail.com

Received: October 24, 2019 | Published: November 22, 2019

\section{Introduction}

Acute appendicitis (AA) is the most common surgical urgency in children, with a developing lifespan risk of $8.6 \%$ in males and $6.7 \%$ in females. The classical pattern of symptoms occurs only in about $50-60 \%$ of cases. Literature reported that rates of appendiceal complication vary depending on age: in overall pediatric population $17-33 \%, 10-20 \%$ in the $10-17$-year-old group, up to $80-100 \%$ in children younger than 4years. Complications increase mortality from $0.002 \%$ to $3 \%$ and morbidity unnecessary appendectomy is reported in $15-40 \%$. A rate of $10-20 \%$ negative appendectomies is considered acceptable and is justified by a surgical approach to the equivocal cases in order to reduce appendiceal complication. Nevertheless, appendectomy is a procedure performed under general an aesthesia and even an unnecessary appendectomy accounts a complication rate of $3-15 \%$, with $2 \%$ of cases requiring a reoperation. ${ }^{1}$ Antibiotic therapy for acute uncomplicated appendicitis is effective in adult patients, but its application in pediatric patients remains controversial. ${ }^{2,3}$ It seems that an in-depth understanding of the pathogenesis of AA will help improve the diagnosis and treatment of this disease.

The state of the problem. Classically, appendicitis is described as a dynamic disease process that comprises 5 stages over a 24-36 hours period. (1) The primary pathogenetic event in most patients with AA is luminal obstruction. This may result from a variety of causes, which include fecaliths, lymphoid hyperplasia, foreign bodies, parasites, and both primary and metastatic tumors. (2) During the second stage, stimulation of the 8th-10th visceral afferent thoracic nerves cause a mild to moderate peri-umbilical pain typically lasts from 4-6hours. (3) As intraluminal pressure increases, appendiceal wall perfusion decreases due to arterial insufficiency. This third stage results in tissue ischemia and mucosal compromise. (4) In the fourth stage bacteria are then able to invade the luminal wall, leading transmural inflammation. (5) At this final stage transmural inflammation extends beyond the appendix, and the parietal peritoneum and adjacent structures also become inflamed. At this stage the pain is more severe, continuous, and often associated with anorexia, fever, nausea and vomiting. ${ }^{4,5}$

If at the beginning of the disease a sudden recanalization of the obstruction zone occurs spontaneously or as a result of antibiotic treatment, the inflammatory process may undergo a reverse development. ${ }^{6}$ A rate of negative appendectomy (i.e., appendectomy performed after a false-positive diagnosis of AA) as high as $25 \%$ in children has been considered an acceptable means of preventing high perforation rates. ${ }^{4}$

In recent years, the frequency of recurrent and chronic appendicitis is described on average as $10 \%$ and $1 \%$, respectively, of the total number of patients with appendicitis. ${ }^{7,8}$ Almost all patients with recurrent or chronic appendicitis can recall at least one episode of an acute attack of abdominal pain resembling an AA that went through without surgery. This suggests that the patient can be operated about $\mathrm{AA}$, while, in fact, there was an exacerbation of the chronic process. ${ }^{5}$

This theory of the pathogenesis of appendicitis leaves several questions open. Why fecal masses stagnate in the appendix (AX) up to the formation of fecal stones? What causes significant differences in AA frequency by age, sex, season, etc.? How does the digestive tract react to the inflammatory process in the AX? The purpose of this study is to investigate the pathogenesis of acute appendicitis.

\section{Material and methods}

The present work is a review of 5 own studies. ${ }^{9-14}$ devoted to different aspects of the etiology and pathogenesis of AA. It analyzes 
the medical history of 3282 children aged 0.5 to 15 years who applied to the Belarusian Children's Surgery Center with suspicion of AA in 1970-1998. Patients were divided into 5 groups depending on the method of study.

The first group consisted of 2932 patients hospitalized with suspected AA. We performed a statistical analysis of the results of histological studies of the AX, depending on the age, gender, season, time of day and the presence of menstruation. The census conducted in the same year made it possible to calculate the actual incidence of AA in different age groups. This was also facilitated by the fact that the children's surgical center was the only institution providing emergency surgical care to children in Minsk. ${ }^{9}$

The second group consisted of 100 appendixes removed during appendectomy. They were selected by the blind method. The X-ray examination was done both without contrast medium and after injection of the barium into their lumen. ${ }^{10}$

The third group consisted of 111 patients admitted from January 1984 to August 1985 in whom the clinical symptoms of AA were doubtful. To clarify the diagnosis the barium enema was accomplished. ${ }^{11}$

The fourth group includes 52 patients aged 7-10 years with complaints of pain in the right ileal region, in which anal canal pressure was measured, according to the method described by us earlier. ${ }^{12}$ The obtained data were compared with the results of anal manometry of 20 children of the same age who entered the hospital for scheduled operations (control group). ${ }^{13}$

Statistical analysis was performed by the method of the Student's $\mathrm{t}$-test. The level of significance was set as $\mathrm{P}<0.05$.

The fifth group consisted of 87 patients aged 7-15years, hospitalized with suspected AA. The radiograph of the abdomen was done in an upright position 10 minutes after taking $50 \mathrm{ml}$ of warm barium. On the radiographs, we determined the areas of the stomach and its gas bubble, their perimeters, the shape factors (the degree of difference of the measured area with the area of the circle), and the maximum and minimum diameters. The contours from the radiographs were taken with the help of a coordinate graph with subsequent computer statistical analysis. ${ }^{14}$

\section{Results}

The first group. Out of 2832 hospitalized patients with suspected AA, 1694 children were operated (7 appendectomies per 1000 child population -7 promille $\mathrm{ppm}$ ). The incidence increased from 2.8 $\mathrm{ppm}$ at the age of $0-4$ years to $8.8 \mathrm{ppm}$ at $5-9$ years and $10.8 \mathrm{ppm}$ at 10-15years. A more detailed analysis revealed a sharp increase in the incidence at 6years. From 6 to 8years, it practically did not change, further increased with a peak of 10-11 years, and then incidence decreased quite sharply (Figure 1). Similar graphs were obtained in a separate analysis by the sex. The second peak of frequency increase in girls was observed at 10 years and in boys at 11 . The same graphs with small fluctuations were characteristic of both destructive and non-destructive (catarrhal) forms of AA, as well as for non-operated patients in whom AA was excluded. At the age of 6 and 9years, the frequency of enlarged lymph nodes in the mesentery increased, especially in girls. A sharp increase in the incidence was in FebruaryMay and September. The lowest incidence rate was in the summer months (June-July), when the incidence was more than 2 times lower than in February.

In daily analysis of the time of occurrence of abdominal pain, two peaks were found: at 7-10 and 16-21hours. In 125 consecutive girls aged 9-15years, the incidence of abdominal pain from the onset of the last menstruation was recorded. In the first days after menstruation, the incidence was minimal. It increased sharply by the 15 th day of the cycle, after which it decreased somewhat and increased again after 28 days or more, i.e. after the delay (Figure 2).

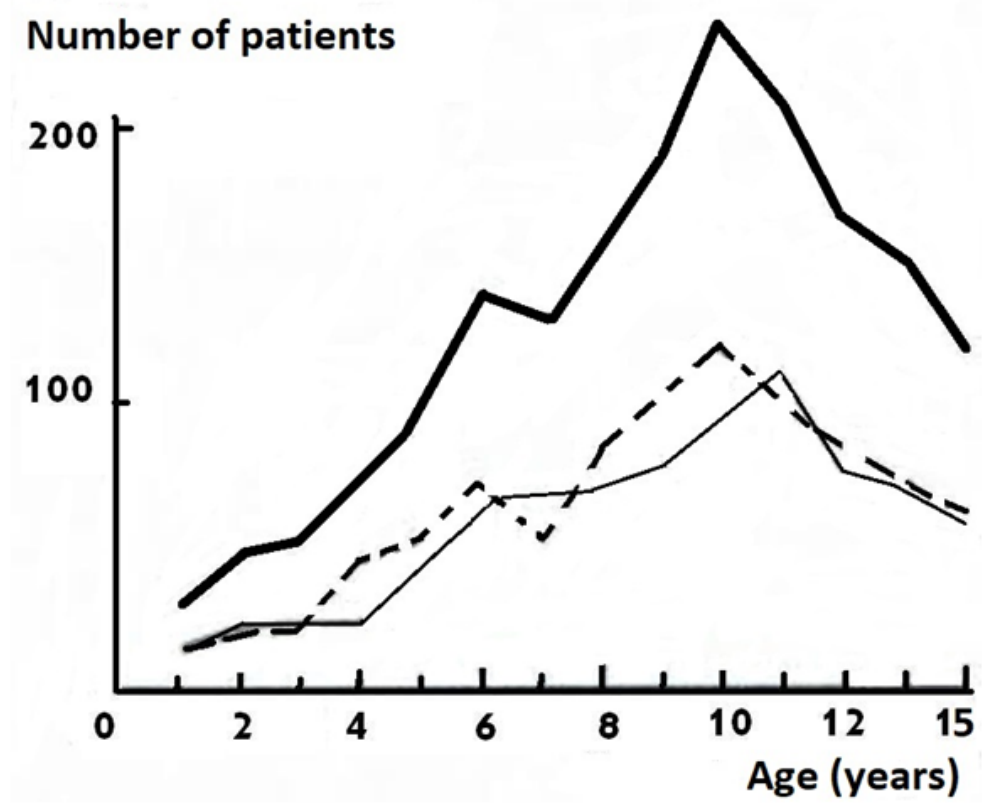

Figure I Dependence of frequency of acute appendicitis on age and sex. Legend: total number, males, - - - females. 


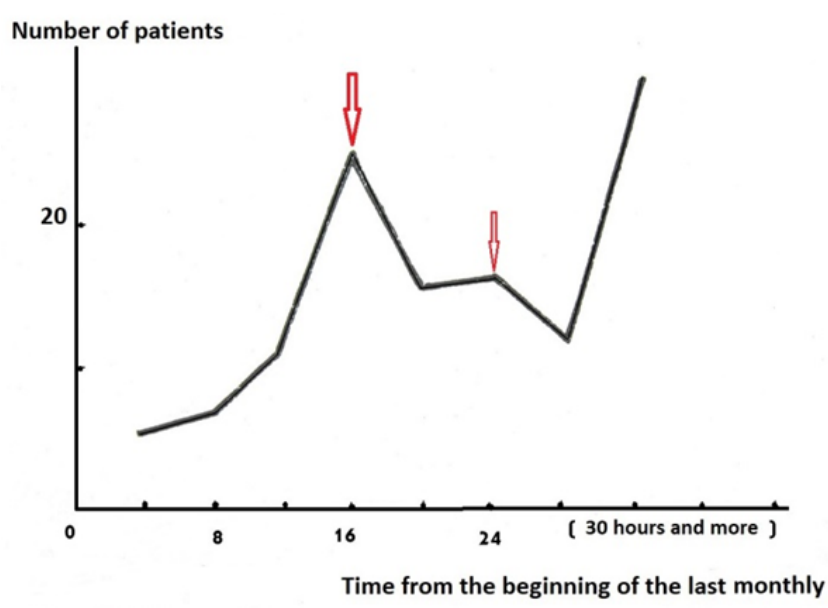

A
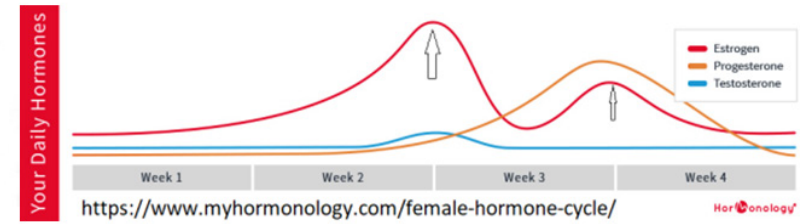

B

Figure 2 Dependence of frequency of AA on time past after the last menstruation.

(A). An increase in the frequency of acute appendicitis. (B) These peaks coincide with to two peaks of the estrogen excretion.

The second group. In 65 out of 100 remote appendices by macroscopic study was established destructive AA, and in 35 cases was the so-called "catarrhal" or "simple" appendicitis. The results of measurements on radiographs are given in Table 1.

All results in the "simple appendicitis" group were the same both in the presence of histological signs of inflammation, and without it. The wall thickness averaged $0.15 \mathrm{~cm}$. This allowed us to estimate the data obtained in this whole group as a norm. The lumen of the AX was passable and uniform throughout its entire length (Figure 3A).

The width and its lumen at the distal part of the AX in destructive AA was significantly wider than in norm. The thickness of the wall is on average $0.25 \mathrm{~cm}$. The contrast stones were found in 8 cases, lowcontrast - in 12, X-ray negative - in 8, disparate contrast inclusions - in 10, foreign bodies - in 2 and unformed stools 2 observations (Figure $3 \mathrm{~B} \& \mathrm{C}$ ). Thus, fecal stones and foreign bodies were found in 42 $(65 \%)$ of 65 patients with destructive AA. As a rule, the width of the stones was much larger than the width of the lumen of the cranial part of the AX. In 20 observations, despite the absence of any inclusions, the width of the closed cavity was much wider than the norm, or, despite the use of high pressure, it was impossible to introduce a contrast agent into the zone of the greatest inflammation. The relief of the mucosa in them was rough, indicating sclerotic changes (Figure $3 \mathrm{D})$. Only in $3(5 \%)$ observations with phlegmonous appendicitis the PX did not differ from normal ones.

The third group. In 78 (70\%) of 111 patients AA was excluded based on barium enema. The lumen of $\mathrm{AX}$ was equally narrow with length at least $6 \mathrm{~cm}$. Its contours were parallel and it itself was convoluted (Figure 4A). The dome of the cecum had convex contours, and its height corresponded to the age norm, which we established in the previous study. ${ }^{12}$

X-ray signs of destructive appendicitis (Figure 4). (A). The appendix is uniformly and deeply filled with barium. Acute appendicitis is excluded. (B). Concavity of the inner part of the dome of the cecum. Ileum and sigma are located away from the cecum. An inflamed omentum takes place between them. This is a symptom of the "window". The diagnosis of AA is confirmed by surgery. (C). The inner contour of the cecum is slightly concave and uneven. Spasm of sigma located opposite the cecum, and symptom of the "window" indicate the presence of inflammation between cecum and sigmoid colon. (D). Concavity of the inner contour of the cecum in combination with a sharp shortening of the dome. Spasm of the terminal ileum and symptom of the "window" make the diagnosis of AA obvious.

In 4 out of 78 cases where AA was excluded, appendectomy was performed, but pathology in the AX was not found. In 21 (19\%) patients, based on the data of barium enema a conclusion was made about the presence of AA. In 20 of them, a destructive appendicitis was revealed on the surgery. In one patient, the abdominal pain disappeared after the barium enema, which made it possible to abandon the operation.

The conclusion about the presence of AA was given based on the detection of the following radiological symptoms. In 16 patients, the AX was not detected. In 4 cases, the contrast medium penetrated into the AX at a depth of 1.1-3.0 cm. In these cases, the internal contours of the AX were uneven, and the AX itself was straightened, which indicates the tension of the tissues. More reliable were the symptoms from the dome of the cecum. In 14 patients there was a pronounced concavity of the inner contour of the dome of the cecum, which is considered a convincing symptom of AA (Figure 4B \& C). ${ }^{15}$ In 5 children this concavity was not, but we noticed that the height of the dome of the cecum was significantly less than the norm (Figure 4D). In addition, in 8 cases, a sharp narrowing of the sigmoid colon located near the AX was found. In 6 cases, a spasm of the terminal ileum was determined. Normally, sigmoid and small intestine are superimposed on the dome of the cecum. At AA, between these intestinal segments a space was detected - the "symptom of the window".

In $12(11 \%)$ patients, the radiographic data were inconclusive. Although the AX was not filled with contrast medium, the dome of the cecum and the adjacent intestinal sections had normal dimensions and configuration. The eight patients were operated. In 2 patients a destructive appendicitis was revealed, in 2 a chronic process was detected. At 2 there was a simple appendicitis, and in 2 observations of inflammatory changes in the appendix was not found. 
Table I Results of X-ray studies of appendices (AX)

\begin{tabular}{lccc}
\hline Parameters $(\mathbf{c m})$ & non-destructive AA & destructive AA & P \\
\hline Length of AX & $7.62 \pm 0.24$ & $7.60 \pm 0.20$ & $>0.2$ \\
Width of proximal part & $0.61 \pm 0.01$ & $0.72 \pm 0.01$ & $<0.001$ \\
Width of distal part & $0.61 \pm 0.01$ & $1.07 \pm 0.03$ & $<0.001$ \\
Width of proximal lumen & $0.35 \pm 0.02$ & $0.38 \pm 0.02$ & $>0.2$ \\
Width of distal lumen & $0.31 \pm 0.02$ & $0.59 \pm 0.01$ & $<0.001$ \\
\hline
\end{tabular}

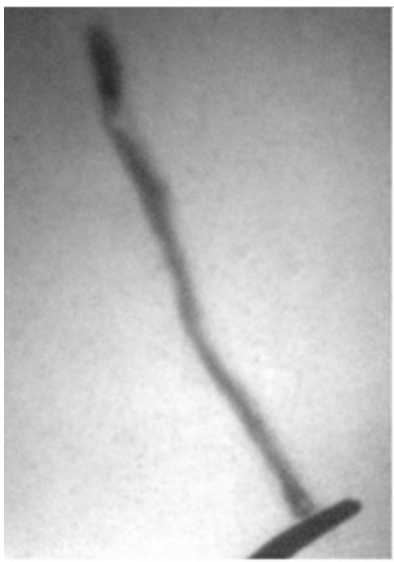

A

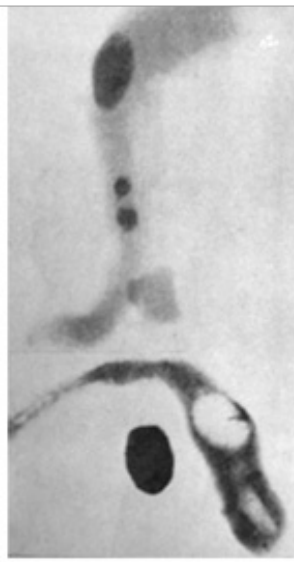

B

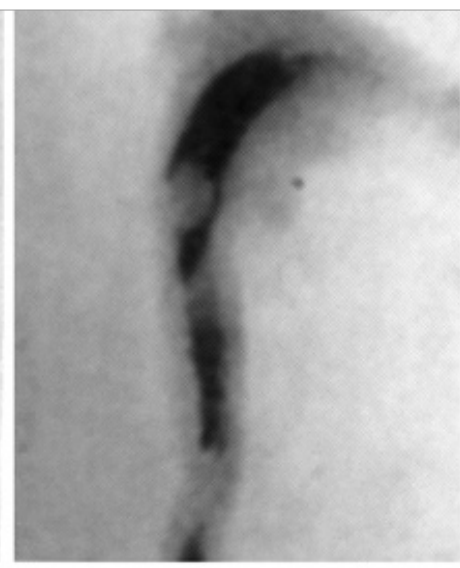

C

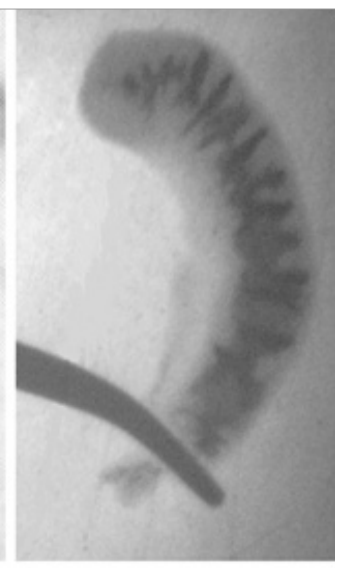

D

Figure 3 Radiographs of appendixes. (A). Radiograph of unchanged appendix uniformly filled with barium. (B). In two destructive AX are the radiocontrast (calcified) fecal stones. The parts of AX located distal to fecal stones are widened. In the lower $A X$, after opening the lumen two cavities, contrasted by air, are determined. Contrasting fecal stone lies next to the AX.(C). The oval X-ray-negative formation (fecal fragment) occludes the enlarged cavity at the apex of the AX. (D). Radiograph of destructive AX without foreign inclusions, but with an extended lumen filled coarsely modified mucosa.

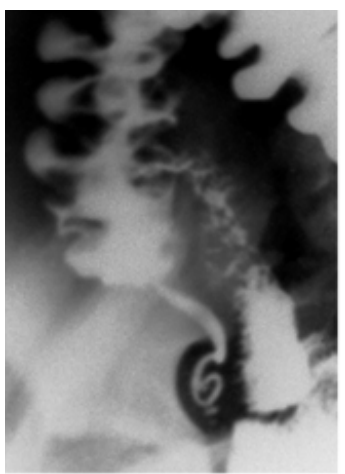

A

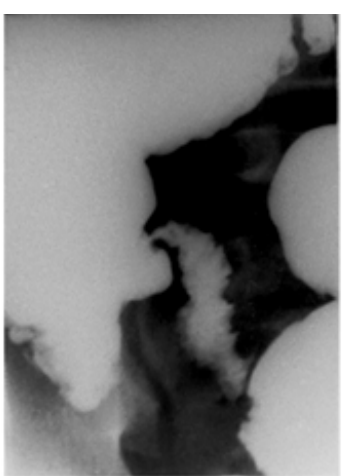

B

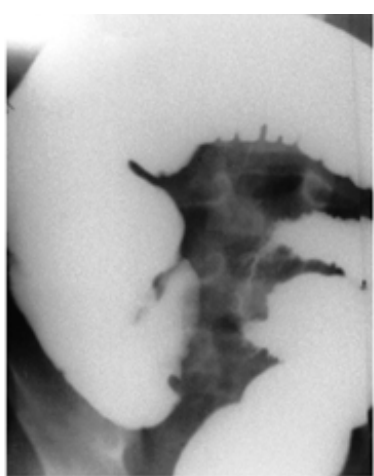

C

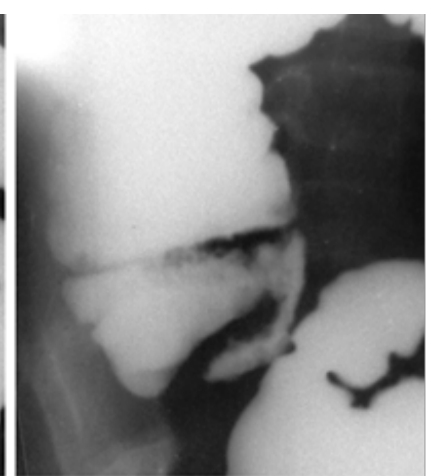

D

Figure 4 X-ray signs of destructive appendicitis. (A). The appendix is uniformly and deeply filled with barium.Acute appendicitis is excluded. (B). Concavity of the inner part of the dome of the cecum. lleum and sigma are located away from the cecum.An inflamed omentum takes place between them. This is a symptom of the "window". The diagnosis of AA is confirmed by surgery. (C). The inner contour of the cecum is slightly concave and uneven. Spasm of sigma located opposite the cecum, and symptom of the "window" indicate the presence of inflammation between cecum and sigmoid colon. (D). Concavity of the inner contour of the cecum in combination with a sharp shortening of the dome. Spasm of the terminal ileum and symptom of the "window" make the diagnosis of AA obvious.

The fourth group. In children of the control group, immediately after the tube was lowered into the anal canal, the pressure was raised to $80-100 \mathrm{~mm} \mathrm{Hg}$. This so-called reflex pressure is caused by the reflex contraction of the external anal sphincter and puborectalis muscle in response to the stretching of the anal canal wall. Within $0.5-1 \mathrm{~min}$, the pressure progressively decreased and was established stably in the range $43.0 \pm 0.8 \mathrm{~mm} \mathrm{Hg}$. This pressure, called basal pressure, is mediated by a tonic contraction of the internal anal sphincter. After injecting $50 \mathrm{~cm}^{3}$ of air into the rectum through the airway channel of the endotracheal tube, a slight increase in pressure to $55-60 \mathrm{~mm} \mathrm{Hg}$ was immediately observed. We called it reactive pressure. After this, the pressure slowly declined by $10-15 \mathrm{~mm} \mathrm{Hg}$. below the basal level with a gradual recovery to the basal level for 4-11 seconds. The dropin pressure below the basal level is due to the reflex relaxation of the internal anal sphincter - an anorectal inhibitory reflex (Figure 5).

In 19 out of 52 children with complaints of pain in the right iliac region, the diagnosis of AA was excluded (subgroup A). In 15 (45\%) of the 33 operated children, a simple AA (subgroup B) was found. In $15(45 \%)$ patients, destructive AA (subgroup B) was found. In three cases, no pathological changes in the AX are found (Table 2). 


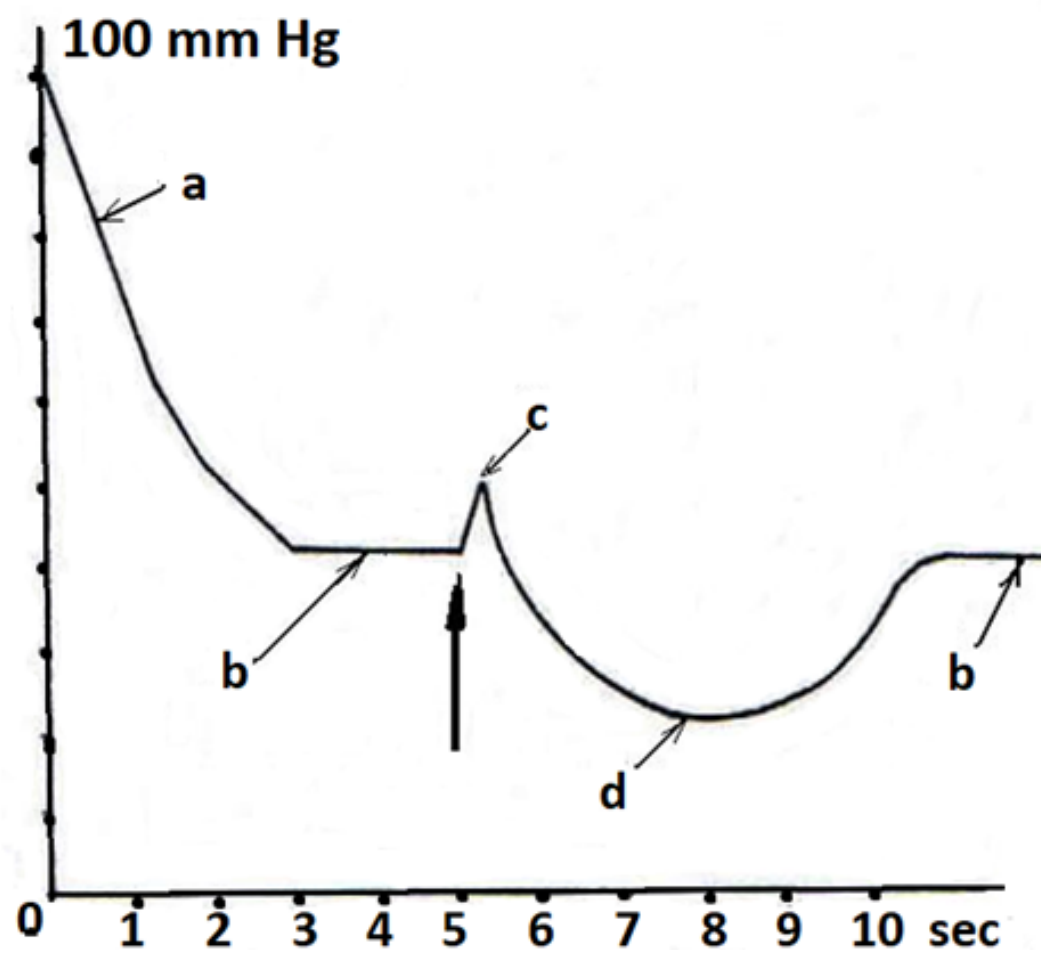

Figure 5 Pressure profile for anal manometry. a. reflex pressure; b. basal pressure; c. reactive pressure $\mathbf{d}$. anorectal inhibitory reflex. A large arrow indicates the moment of air injection into the rectum.

Table 2 Results of an anal canal manometry in children with suspected AA

\begin{tabular}{lcccc}
\hline Anal canal pressure & Subgroup A (I) & Subgroup B (2) & Subgroup C (3) & P \\
\hline Basal pressure & $53.6 \pm 1.1$ & $69.3 \pm 1.7$ & $73.0 \pm 3.4$ & $\begin{array}{l}\text { P I-2,3<0.00I } \\
\text { P } 2-3>0.2\end{array}$ \\
Reactive pressure & $58.5 \pm 2.5$ & $91.0 \pm 2.7$ & $111.3 \pm 5.8$ & $\begin{array}{l}\text { P I-2,3<0.00I } \\
\text { P } 2-3<0.01\end{array}$ \\
\hline
\end{tabular}

Only basal and reactive pressures were significantly higher in operated patients, both with simple and destructive AA, compared with non-operated children. With destructive AA, the pressure was higher than with a simple one. But statistically significant was the increase in only the reactive pressure.

Fifth group. In 30 out of 87 children with suspected AA the destructive appendicitis was diagnosed after the operation. In 14 cases, superficial inflammation was detected. The diagnosis of AA was excluded in remaining 43 patients, and they were discharged without surgery. All the parameters, including the area of the stomach and its gas bubble, their perimeters, shape factors, as well as the maximum and minimum diameters with high reliability $(\mathrm{P}<0.001)$ were less with destructive appendicitis compared with unoperated children. Differences between the parameters of patients with destructive and simple AA were not significant $(\mathrm{P}>0.5)$.

\section{Discussion}

A sharp increase in the frequency of AA at 6,11 and 25 years, as well as the difference in sex completely correlate with the excretion of sex hormones. Up to 7years, the concentration of estrogens in children of both sexes is the same. Estrogen excretion increases dramatically at 5-7years, then growth slows somewhat and again increases in female to $10-11$, and in male to $12-13$ years with a maximum in the third decade. ${ }^{16,17}$ After 7years, the amount of estrogen in female increases more than in male with a one-year lag in male as in AA. ${ }^{18}$ Similar changes are also described with respect to androgens, folliclestimulating and luteinizing hormones. ${ }^{19}$ Meanwhile, the increase in the frequency of AA in males 10-11years old coincides with the decrease in the concentration of serum cortisol. ${ }^{20}$

In our study, there was a significant increase in incidence AA in the middle of the monthly cycle, which also coincides with an increase in estrogen excretion..$^{21}$ In modern literature, estrogens are considered as amplifiers of cellular proliferation and humoral immune response..$^{22}$ Thus, the influence of sex hormones, including estrogen on the development of AA in children is not in doubt. Probably, hormones are one of the triggers of the volumetric increase of the epithelial and lymphoid tissues of the AX. Given that the absolute level of sex hormones in children is lower than in adults, the observed dependence is not due to the absolute level, but to a sharp rise in the excretion of sex hormones.

As shown by our X-ray studies of the AX, in the vast majority of cases of destructive appendicitis, the lumen in the distal part of the AP is expanding. This is due to obstruction in the more proximal part of the AP and the formation of a closed cavity with high pressure in 
it. In $42(65 \%)$ of 65 cases, obstruction of the lumen appeared due to overlapping of the lumen by fecal stones and foreign bodies. In $16(25 \%)$ cases, the expansion of the lumen of the AX indicated that there was a closed cavity with high pressure. In $4(6 \%)$ cases, in spite of the use of high pressure, it was not possible to fill the AX with a contrast agent, which implies obstruction or obliteration of the AX lumen, and only in $3(4 \%)$ there were no signs of formation of a closed cavity in cases with a histological conclusion of the phlegmonous inflammation.

All inclusions (fecal stones, foreign bodies) were much wider than the lumen of the proximal part of the AX. This suggests that they could not penetrate the AX on the eve of an acute attack. Symptoms of the disease appear soon after the onset of obstruction of the lumen. But it is obvious that feces or foreign bodies were stuck there, increased in size and had time to be encrusted with calcium long before the appearance of complete obstruction. This is also evidenced by expressed sclerotic changes in the wall of the AX. The mere fact that the liquid chyme that is ingested in the AX from the ileum was not expelled by the peristaltic wave indicates a serious damage to the neuromuscular function of the AX before the appearance of fecal masses. Di Sebastiano with co-authors, applied special methods of studying of AX, where there were no signs of inflammation in the usual histological examination. They found neuroproliferation in combination with an increase in neurotransmitters SP and VIP, which could be the cause of pain in the right ileal region. The authors suggested that as a result of the interaction of the nervous and immune systems, neuroimmune appendicitis appears as a separate pathological entity. ${ }^{23}$

Some researchers believe that destructive appendicitis in many cases is the culmination of a process that unfolds as a result of changes that have occurred in the AX in previous less severe attacks. Repeated attacks AA intensify the phenomenon of sclerosis, right up to the obliteration of the AX lumen. ${ }^{24}$ When in the AX develops hyperplasia of the mucosa, mainly its lymphoid elements, as a result of an immunological reaction to a viral infection or an allergic process, this leads to a sharp narrowing of the lumen of the AX. If the complete obstruction of the AX has not occurred, the inflammation undergoes reverse development, but as a result of damage to the nervous apparatus, the peristalsis of the AX is impaired, and scar changes remain in the mucosa that disrupt the normal emptying of the AX. This contributes to the retention of feces in it. They eventually become denser, increase in volume and are encrusted with salts. Repeated immunological reaction of the mucosa against the background of coprolites and / or scars leads to obstruction of the AX and development of AA in the stages described above.

The concavity of the inner contour of the cecum, the narrowing of the lumen of the sigmoid colon and ileum during barium enema cannot be explained by the volume mass pressure, as some researchers have claimed. ${ }^{25}$ We noticed that in some patients with AA, the dome of the cecum is sharply shortened. If normally the height of the dome of the cecum is equal to or longer than the width, then with AA the height of the dome may be 2 times shorter than its width. With intra-operative verification of radiologic symptoms, we found that these signs do not depend on the volume of the AX, which may be out of contact with these parts of the intestine. We believe that these symptoms are due to an increase in the tone of the smooth muscle of these parts in response to the irritation emanating from the inflammatory focus. ${ }^{11}$ Normally, the ileum and sigmoid colon are located near the cecum. We noticed that in OA, the distance between the cecum and intestines increases significantly, which is probably due to the location of the inflamed momentums in this area (window symptom).

We also suggested that increased tonus may extend to other parts of the digestive tract. Since the internal anal sphincter is a thickened continuation of the circular layer of the colon, it can be concluded that its tone reflects the tonic state of the gut. We measured anal pressure in 52 children aged 7-10 years with complaints of pain in the right ileal region. Basal and reactive anal pressures were significantly higher $(\mathrm{P}$ $<0.001$ ) in patients operated on acute appendicitis, compared to nonoperated children. In destructive appendicitis, the pressure was higher than with a simple one. But this difference was significant $(\mathrm{P}<0.02)$ only for reactive pressure. ${ }^{13}$

We concluded that the inflammatory process in AA is accompanied by an increase in basal pressure in the anal canal and an increase in its reactivity to irritation of the rectum in most patients. The described phenomenon is not strictly specific for AA. At the same time, the more pronounced the inflammatory process, the higher the anal pressure.

At this stage, there were reasons to believe that with AA, the stomach tone also increases. On the radiographs of the stomach with barium, a highly reliable decrease in the area of the stomach and the gas bubble, their perimeters, shape factors, as well as the maximum and minimum diameters in destructive AA were found compared to with non-operated children. Our results indicate an increase in the tone of the stomach in AA. In this way, the appearance of vomiting and pain in epigastrium in the first hours of the disease can be explained by the contraction of the stomach, as a result of increasing its tone.

\section{Conclusion}

Based on the analysis of literature and our own research, we came to the following understanding of the etiology and pathogenesis of acute appendicitis. The lumen of the AX sharply narrows as a result of the immunological reaction with an increase in the volume of its lymphoid tissue. This reaction may occur in response to an inflammatory process in the lymphoid tissue in another place, a viral infection, or an allergy. It is more likely with an increase in the level of sex hormones. In some number of cases the lumen of the AX completely overlaps, so that a closed cavity in the distal part of the $\mathrm{AX}$ is formed.

Since the mucosa continues to produce mucus, the pressure in the enclosed cavity rises and it expands. At certain values within the luminal pressure, the arterial perfusion of the wall of the AX is reduced. This leads to tissue ischemia and impaired mucosal protective function. Probably, at this stage the intra-abdominal pressure reflexively increases and the tonus of the whole digestive tract and especially its sphincters rises. Increased pressure in the stomach with a simultaneous increase in the tone of the pyloric sphincter, cause epigastric pain and vomiting. Due to the violation of the epithelial mucous barrier, bacteria enter the AX wall with the appearance of transmural inflammation, which passes to the parietal peritoneum of the $\mathrm{AX}$ and adjacent organs.

Closing of the AX lumen with hypertrophic mucosa is sometimes not enough strong. A closed cavity can open with recovery of the lumen continuity of the AX. However, the inflammatory reaction leaves sclerotic measurements that violate emptying of the AX. Sometimes hyperplasia of the mucosa occurs without a complete overlap of the AX lumen and may even be so insignificant that it does not cause clinical symptoms. However, after its resolution remains a varying degree of damage to the nervous system, leading to impaired peristalsis of the AX is disrupted. The feces are delayed in in the AX. 
They thicken, increase in diameter and are encrusted with salts. In one of the subsequent attacks of hyperplasia, the thickened wall of the AX enfolds a stone, which is pressed into the wall of the AX with force. In such cases, necrosis of the wall with perforation of the AX quickly arises.

Thus, AA in most cases is an acute inflammatory process that has developed against the background of previous attacks, which cause development scars, damage to the nervous system of the AX, and therefore, a violation of its peristaltic function. As our studies have shown, inflammation in the AX causes an increase in the tone of the stomach, anal canal and an increase intra-abdominal pressure. These facts give reason to assume that with acute appendicitis, the tone of all parts of the digestive tract increases. Probably, the tone of the intestinal segments located near the $\mathrm{AX}$ increases to a greater extent than tone of the remote segments. This is manifested by concavity of the internal contour of the cecum, i.e. zone, where the base of AX is located, shortening of the dome of the cecum, by spasm the terminal section of the ileum and sigmoid colon which located near AX. These symptoms can be used to diagnose chronic appendicitis, as well as in the diagnosis of OA using CT or MRI.

\section{Acknowledgments}

None.

\section{Conflicts of interest}

Author declares there are no conflicts of interest.

\section{References}

1. Cesare AD, Parolini F, Morandi A, et al. Do we need imaging to diagnose appendicitis in children? Afr J Paediatr Surg. 2013;10(2):68-73.

2. Svensson JF, Patkova B, Almström M, et al. Nonoperative treatment with antibiotics versus surgery for acute nonperforated appendicitis in children: a pilot randomized controlled trial. Ann Surg. 2015;261(1):6771.

3. Huang L, Yin Y, Yang L, et al. Comparison of Antibiotic Therapy and Appendectomy for Acute Uncomplicated Appendicitis in Children: A Meta-analysis. JAMA Pediatr. 2017;171(5):426-434.

4. Brennan GDG. Pediatric appendicitis: pathophysiology and appropriate use of diagnostic imaging. CJEM. 2006;8(6):425-432.

5. Birnbaum BA, Wilson SR. Appendicitis at the millennium. Radiology. 2000;215(2):337-348.

6. Cobben LP, de Van OtterlooAM, Puylaert JB. Spontaneously resolving appendicitis: frequency and natural history in 60 patients. Radiology. 2000;215(2):349-352.

7. Hollerman JJ, Bernstein MA, Kuttamasu SR, Sirr SA. Acute recurrent appendicitis with appendicolith. Am J Emerg Med. 1988;6(6):614-617.

8. Hawes AS, Whalen GF. Recurrent and chronic appendicitis: the other inflammatory condition of the appendix. Am Surg. 1994;60(3):217-219.
9. Levin MD. [Role of hormonal factor in the pathogenesis of acute appendicitis]. Zdravoochranenie Belarusii; 1984;46(11):32-36.

10. Levin MD, Shuan SI. [Role of process vermiformis impaction and foreign bodies in the development of acute appendicitis]. Zdravoochranenie Belarusii. 1985;(12):31-36.

11. Misharev OS, Levin MD, Shuan SI. [X-ray diagnosis of acute appendicitis by measured hydrostatic irrigoscopy]. Khirurgiia (Mosk). 1986;(8):61-65.

12. Levin MD. [Roentgenologic anatomy of the colon and rectum in children]. Vestn Rentgenol Radiol. 1985;(2):40-45.

13. Levin MD, Misharev OS, Al'khimovich VN. [Anal manometry in acute appendicitis]. Klin Khir. 1986;(6):35-38.

14. Levin MD, Khomich VM, Nalibotskiǔ BV. [The possibility of $\mathrm{x}$-ray diagnosis of acute appendicitis in children]. Klin Khir. 1989;(6):38-40.

15. Hatch EI Jr, Naffis D, Chandler NW. Pitfalls in the use of barium enema in early appendicitis in children. J Pediatr Surg. 1981;16(3):309-312.

16. Stárka L, Hoza J, Hampl R, Straková M. [Level of plasma androgens during the course of puberty in boys]. Cesk Pediatr. 1976;31(1):11-14.

17. Bednyakova LS. Age dynamics of excretion of pituitary gonadotropins and ovarian steroids with urine in normally developing girls. In the book: 2nd All-Union Biochem Congress, Tashkent, Section 5. 1969. p.72-73.

18. Bildingmaier F, Nagner-Barnack M, Butenandt $\mathrm{O}$, et al. Plasma estrogen in childhood and puberty under physiologic and pathologic conditions. Pediatr Res. 1973;7(11):901-907.

19. Sizonenko PC, Paunier L. Hormonal changes in puberty III: Correlation of plasma dehydroepiandrosterone, testosterone, $\mathrm{FSH}$, and $\mathrm{LH}$ with stages of puberty and bone age in normal boys and girls and in patients with Addison's disease or hypogonadism or with premature or late adrenarche. J Clin Endocr. 1975;41(5):894-904.

20. Apter D, Pakarinen A, Hammond GL, et al. Adrenocortical function in puberty. serum ACTH, cortisol and dehydroepiandrosterone in girls and boys. Acta Pediatr Scand. 1979;68(4):599-604.

21. Arnbjörnsson E. The influence of oral contraceptives on the frequency of acute appendicitis in different phases of the menstrual cycle. Surg Gynecol Obstet. 1984;158(5):464-466.

22. Cutolo M, Brizzolara R, Atzeni F, et al. The immunomodulatory effects of estrogens: clinical relevance in immune-mediated rheumatic diseases. Ann N Y Acad Sci. 2010;1193(1):36-42.

23. Di Sebastiano P, Fink T, di Mola FF, et al. Neuroimmune appendicitis. Lancet. 1999;354(9177):461-466.

24. Gorenstin A, Serour F, Katz R, et al. Appendiceal colic in children: a true clinical entity. J Am Coll Surg.1996;182(3):246-250.

25. Hatch EI Jr, Naffis D, Chandler NW. Pitfalls in the use of barium enema in early appendicitis in children. J Pediatr Surg. 1981;16(3):309-312. 